\title{
Probing the Earth Core Composition with Neutrino Oscillation Tomography
}

\author{
Simon Bourret \\ Université de Paris, APC, CNRS, CEA, Observatoire de Paris, F-75006 Paris, France \\ E-mail: simon.bourret.fr@gmail.com
}

João A. B. Coelho

Laboratoire de l'accélérateur Linéaire, Univ. Paris-Sud, CNRS/IN2P3, Univ. Paris-Saclay,

France

E-mail:coelho@lal.in2p3.fr

\section{Edouard Kaminski}

Université de Paris, Institut de physique du globe de Paris, CNRS, F-75005 Paris, France

E-mail:kaminski@ipgp. fr

\section{Véronique Van Elewyck ${ }^{*}$}

Université de Paris, APC, CNRS, CEA, Observatoire de Paris, F-75006 Paris, France

E-mail: elewyckeapc.in2p3.fr

\begin{abstract}
Atmospheric neutrinos open the way to alternative probing methods to study the structure and composition of the inner Earth, complementary to geophysical methods. At GeV energies, the flavour oscillations of neutrinos crossing the Earth are distorted due to coherent forward scattering on electrons along their path. The signature of these matter effects in the neutrino angular, energy and flavour distributions may provide sensitivity to the electron density, and thus the composition, in the different layers traversed.

The combination of this neutrino-based measurement with a reference mass density profile constrains the effective proton-to-nucleon ratio of the medium (Z/A). Since this parameter varies among chemical elements, this technique has the potential to provide unprecedented insights into the chemical composition of the core. Current geophysical data require the presence of a few wt $\%$ of light elements inside the iron core, whose nature and amount remain controversial.

Such a measurement requires large-sized neutrino detectors with good efficiency in the relevant energy range and precise determination of the neutrino energy, arrival direction, and flavour. Considering a generic but realistic model of detector response, we discuss the influence of various detector performance indicators on the sensitivity to the average Z/A in the core. Starting from specific examples of the next-generation detectors (ORCA, DUNE), we also identify the main improvements required to reach a measurement of the $\mathrm{H}$ content of the core at the $1 \mathrm{wt} \%$ level.
\end{abstract}

36th International Cosmic Ray Conference -ICRC2019-

July 24th - August 1st, 2019

Madison, WI, U.S.A.

\footnotetext{
* Speaker.

${ }^{\dagger}$ Also at Institut Universitaire de France, 75005 Paris, France.
} 


\section{Introduction}

Our knowledge on the structure and composition of the deep Earth, described as a metallic core surrounded by a silicate shell, is essentially indirect and based on the inversion of seismic data, geodetic measurements and experimental petrology. The mantle, representing the largest fraction of the Earth's volume, comprises an upper (from the base of the crust to about $400 \mathrm{~km}$ depth) and a lower (660-2900 km depth) region, separated by the transition zone. To first order, the mantle is considered to have uniform chemical composition (pyrolite). The Earth's core is believed to be mostly formed of Fe-Ni alloy, and subdivided into a solid inner core with an approximate radius of $1200 \mathrm{~km}$, surrounded by the molten outer core. The combination of seismic data with constraints on the radius, total mass and momentum of inertia can be used to infer the radial matter density profile of the Earth, as described e.g. in the Preliminary Reference Earth Model (PREM) [1] developed in the early '80s. The main features of the PREM profile are illustrated in Fig. 1.

As first proposed in [2], comparison between the PREM model and high-pressure/high-temperature properties of Fe-Ni alloys shows that the density jump at the inner core boundary (ICB) as well as the seismic velocities in the core required the presence of a few percents in weight (wt\%) of light elements. Si, O, S, C and $\mathrm{H}$ (and some of their combinations) are the most popular elements that have been considered so far [3]. But the precise nature and amounts of the light element(s) involved cannot be fully determined based on seismology and high pressure/high temperature experimental petrology or ab initio calculations alone, and models of core composition must rely also on specific scenarios of Earth formation [4]. Amongst the different light elements, $\mathrm{H}$ has received a renewed interest in the past years based on high-pressure/high-temperature experiments that confirmed the possibility to put a significant amount of $\mathrm{H}$ in the core [5]. $\mathrm{H}$ is the most abundant element in the proto-solar nebula, and it has been argued that the incorporation of a few wt $\%$ of $\mathrm{H}$ in the core was possible through the accretion of $\mathrm{H}_{2} \mathrm{O}$-bearing materials [6]. Furthermore, the effect of $\mathrm{H}$ on density and seismic velocities is such that about $1 \mathrm{wt} \%$ of $\mathrm{H}$ in the outer core could be enough to fit PREM [7], which would in turn rule out the presence of other light elements. Up to now however, no method is available to directly constrain the amount of $\mathrm{H}$ in the core.

Because of their weak interactions, neutrinos can offer an alternative way of probing the structure and composition of the inner Earth, complementary to geophysical methods [8]. Atmospheric neutrinos are particularly appealing for such studies as they span a wide range of energies and path lengths across the Earth. At $\sim \mathrm{GeV}$ energies, these neutrinos undergo flavour oscillations which are modified due to coherent forward scattering on electrons [9]. The signature of these matter effects in the angular, energy and flavour distributions of neutrinos detected at the surface may therefore provide sensitivity to the electron density $n_{e}$ in the different layers of matter traversed. The ratio of electron density $n_{e}$ to mass density $\rho_{m}$ scales with the effective proton-to-nucleon ratio (hereafter denoted $Z / A$ ), which depends on the chemical and isotopic composition of the medium:

$$
\frac{n_{e}}{\rho_{m}}=\frac{N_{A}}{m_{n}} \times \frac{Z}{A},
$$

where $N_{A}$ is the Avogadro number and $m_{n}$ the nucleon mass. The combination of a neutrinobased measurement of the electron density with the PREM mattter density profile can therefore constrain the effective proton-to-nucleon ratio of the medium (Z/A) [10]. Since this parameter 


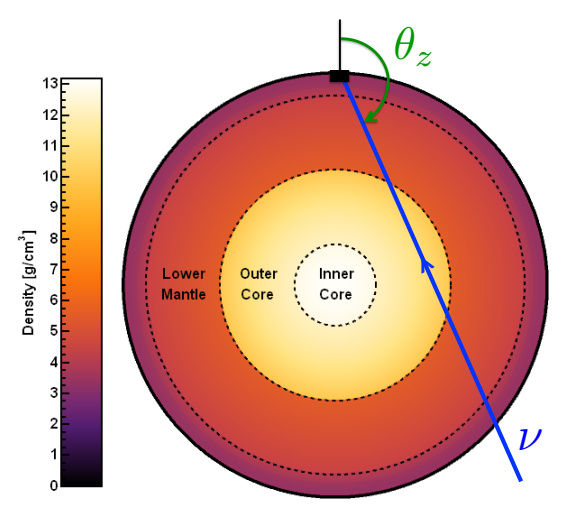

\begin{tabular}{llll} 
Layer & Shells & {$\left[R_{-}, R_{+}\right](\mathrm{km})$} & $\mathrm{Z} / \mathrm{A}$ \\
\hline Inner core & 4 & $0-1221.5$ & 0.4661 \\
Outer core & 20 & $1221.5-3480$ & 0.4661 \\
Lower mantle & 11 & $3480-5701$ & 0.4954 \\
Upper mantle & 5 & $5701-6346$ & 0.4954 \\
Crust & 2 & $6346-6368$ & 0.4956 \\
Water & 1 & $6368-6371$ & 0.5525 \\
Atmosphere & 1 & $6371-6386$ & 0.4991 \\
\hline
\end{tabular}

Figure 1: Left: Terrestrial density profile according to the 44-shells model used in this contribution, also showing the main inner compositional layers. Right: Compositional layers with the associated number of constant density shells, the exact innermost and outermost radius, and the corresponding default $Z / A$ value [14, 15] .

varies among chemical elements, e.g. 0.4655 for $\mathrm{Fe}$ and $1 \mathrm{for} \mathrm{H}$, this technique has the potential to provide unprecedented insights into the chemical composition of the outer core, and in particular its hydrogen content. Different benchmark models of the outer core composition will be discussed in Section 2, and perspectives for disentangling the most extreme ones with the next-generation atmospheric neutrino detectors will be provided in Section 3.

\section{Methodology}

\subsection{Underlying signal}

The presence of electrons in the medium traversed by the neutrinos induces an effective potential for charged-current interactions $V_{C C}= \pm \sqrt{2} G_{F} N_{e}(x)$ (where the \pm apply respectively to $v_{e}$ and $\bar{v}_{e}$ ), which modifies the neutrino oscillation probabilities. The size of this effect depends on the neutrino energy as well as the length of the neutrino path. The analysis therefore relies on the computation of energy $E$ and zenith angle $\theta_{z}$ distributions of atmospheric neutrino events of different flavours.

The model of incoming atmospheric neutrino flux is taken from [11] and the probabilities of neutrino flavour transitions are computed using the OscProb software [12]. For a given incident angle $\theta_{z}$, the trajectory of a neutrino through the Earth is modelled along the corresponding baseline through a sequence of steps of constant electron density. The Earth electron density is a radial model with 44 concentric shells of constant electron density, where mass density values are fixed and follow the PREM. These shells are distributed into seven chemical layers, where the composition, and hence the $Z / A$ factor, are assumed to be uniform (see Fig. 1). In each shell, the electron density is determined from the mass density and $Z / A$ according to eq. (1.1). The quantum evolution equations for the neutrino states are solved numerically for each shell along the neutrino path.

Finally, the rate of events interacting in an hypothetical detector is computed using neutrinonucleon cross-sections weighted for water molecules, obtained with the GENIE Monte Carlo generator [13]. This differential rate (corresponding to the number of neutrino interactions of given flavour, energy $E$ and zenith $\theta$ occurring in the detector per unit exposure) is computed for each 

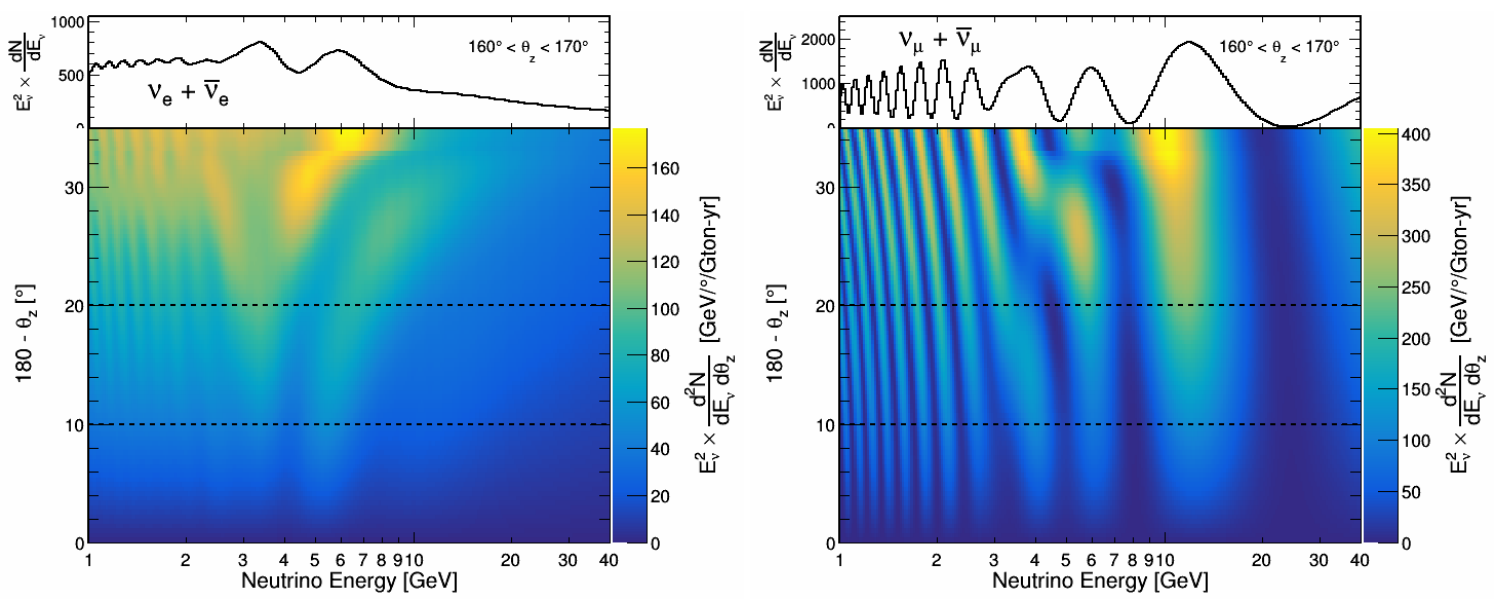

Figure 2: Rate of neutrino interactions as a function of energy and zenith angle, multiplied by $E^{2}$, for $v_{e}+\bar{v}_{e}$ (left) and $v_{\mu}+\bar{v}_{\mu}$ (right), as obtained using the standard compositional model (A) of Table. 1. The upper panels show the corresponding rate profiles integrated over the range of zenith angles $\theta_{z} \in\left[160^{\circ}, 170^{\circ}\right]$, which encompasses the neutrino trajectories that cross the innermost part of the outer core (horizontal band delimited by the two black lines in the lower panels).

(anti-)neutrino flavour $\beta$ as:

$$
\frac{\mathrm{d} N_{\beta}^{\mathrm{int}}(E, \theta)}{\mathrm{d} E \mathrm{~d} \theta}=\sigma_{v_{\beta}}(E) \cdot \sum_{v_{\alpha}} P_{v_{\alpha} \rightarrow v_{\beta}}(E, \theta) \cdot \frac{\mathrm{d} \Phi_{v_{\alpha}}}{\mathrm{d} E \mathrm{~d} \theta}(E, \theta)
$$

where $\sigma_{v_{\beta}}$ is the total interaction cross-section for neutrino type $v_{\beta}, P_{\alpha \rightarrow \beta}$ is the $v_{\alpha} \rightarrow v_{\beta}$ oscillation probability, and $\mathrm{d} \Phi_{v_{\alpha}} / \mathrm{d} E \mathrm{~d} \theta$ is the unoscillated differential flux of atmospheric neutrinos at the detector location. Fig. 2 shows the resulting rates of electron and muon (anti)neutrino flavours obtained for the benchmark compositional model (A) of the outer core (as defined in Table 1).

The impact of different compositional models of the outer core can be studied by investigating the relative difference $(\Delta N / N)$ in the expected number of neutrino events of a given flavour $\left(v_{e}+\bar{v}_{e}\right.$ or $\left.v_{\mu}+\bar{v}_{\mu}\right)$. Fig. 3 shows this quantity as a function of energy, for benchmark models
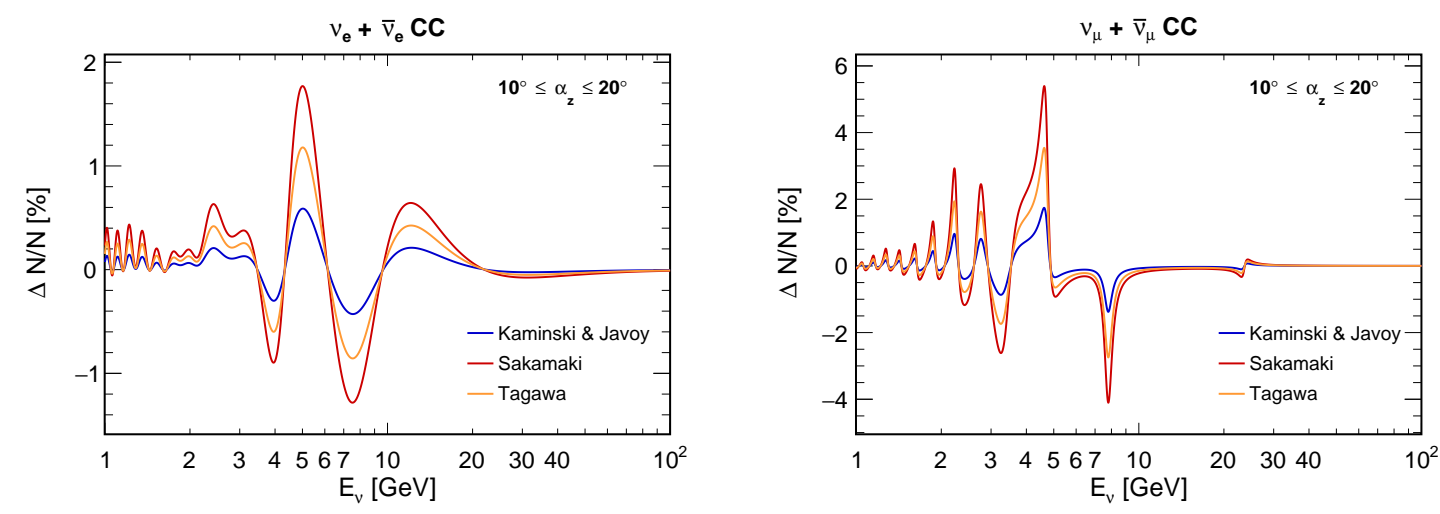

Figure 3: Relative difference $\Delta N / N$ in the number of events occurring in the $v_{e}+\overline{v_{e}}$ (left) and $v_{\mu}+\overline{v_{\mu}}$ (right) channel), for the compositional models described in Table 1, taking Model (A) as a reference. 


\begin{tabular}{lccccc} 
Model & $(0)$ & $(\mathrm{A})$ & $(\mathrm{B})$ & $(\mathrm{C})$ & $(\mathrm{D})$ \\
& Default $(\mathrm{Fe}-\mathrm{Ni})$ & Badro[4] & Kaminski \& Javoy[15] & Tagawa[16] & Sakamaki[5] \\
\hline Light elements & none & $7 \%$ wt Si & $2 \%$ wt Si & $0.3 \%$ wt H & $1 \%$ wt H \\
& & $2 \%$ wt O & $4 \%$ wt O & $6.5 \%$ wt Si & \\
\hline Z/A & 0.4661 & 0.4680 & 0.4689 & 0.4697 & 0.4713 \\
\hline
\end{tabular}

Table 1: Weight fraction of light elements and average $Z / A$ for different compositional models considered in this study. In all models the Ni content is set to $5 \mathrm{wt} \%$ and $\mathrm{Fe}$ is the complement to $100 \%$.

with different weight fractions of light elements in the outer core, taken from the literature. The curves are obtained assuming a perfect detector with infinite exposure (hence the absence of statistical error bars). Both electron and muon channels exhibit fast oscillations in $\Delta N / N$ at low $(<2$ $\mathrm{GeV}$ ) energies, reflecting the small changes in oscillation phase induced by the dependence of the parameters in the electron density model chosen for the outer core. For all models, the underlying signal is most visible in the muon neutrino channel, where it can reach up to 5 or $6 \%$ (depending on the zenith angle) when comparing the extreme compositional models (D) and (A). The two standard compositional models (A) and (B) differ by 1 to $2 \%$ at most for both flavours, suggesting that their disentanglement would be challenging even without considering the effect of finite detector performances.

\subsection{Detector response modeling}

Oscillation tomography requires neutrino detectors with high detection, reconstruction and identification performances in the $1-40 \mathrm{GeV}$ energy range. Such detectors are currently being proposed and built as part of the global neutrino physics program, with the aim of improving the measurement of the oscillation parameters and of solving the questions of the neutrino mass hierarchy and $\mathrm{CP}$ violation in the leptonic sector.

The next generation of water- (and ice-) Cherenkov neutrino detectors includes HyperKamiokande [17], KM3NeT/ORCA [18] and PINGU [19], with instrumented volumes ranging from 0.5 to a few Mtons. The DUNE experiment [20], primarily designed to work in synergy with the LongBaseline Neutrino Facility (LBNF), will consist in large Time Projection Chambers filled with liquid Argon. While more modest in size ( $\sim 50 \mathrm{ktons})$, DUNE is expected to reach an unprecedented precision in the energy and angular reconstruction of neutrino events by disentangling the measurement of the charged lepton and of the hadronic system produced in the neutrino charged-current interaction. Both DUNE and HyperKamiokande claim a sub-GeV detection threshold and almost perfect separation of the electron and muon channels, although for DUNE it is still unclear how these performances will be transferable to atmospheric neutrino signals (without prior knowledge of the arrival direction). The large dimensions of ORCA and PINGU allow them to record 10 to 100 times larger statistics of events than DUNE and HyperKamiokande in a given time span. However, the relative sparseness of their instrumentation limit their event reconstruction and classification performances. Events are classified into track-like (when a $v_{\mu}$-induced muon is reconstructed) and shower-like channels, the latter comprising not only $v_{e}$ but also $v_{\tau} \mathrm{CC}$ interactions and all-flavours $\mathrm{NC}$ interactions.

While preliminary sensitivity studies for Earth tomography have been performed for some of these detectors using detector simulation tools internal to the collaborations [17, 19, 21], we choose to adopt here a more generic and flexible approach based on analytical response functions for the 


\begin{tabular}{|c|c|c|c|c|c|c|c|c|}
\hline Detector & $M_{t o t}$ (Mton) & $E_{t h}(\mathrm{GeV})$ & $E_{p l}(\mathrm{GeV})$ & $\sigma(E) / E$ & $\sigma_{\theta}(\mathrm{deg})$ & $E_{\text {th }}^{\text {class }}(\mathrm{GeV})$ & $E_{p l}^{\text {class }}(\mathrm{GeV})$ & $P_{\max }^{\text {class }}$ \\
\hline ORCA-like [18] & 8 & 2 & 10 & $25 \%$ & $30 / \sqrt{E}$ & 0.1 & 10 & $85 \%$ \\
\hline HyperKamiokande-like [17] & 0.40 & 0.1 & 0.2 & $15 \%$ & $15 / \sqrt{E}$ & 0.1 & 0.2 & $99 \%$ \\
\hline DUNE-like [20] & 0.04 & 0.1 & 0.2 & $5 \%$ & 5 & 0.1 & 0.2 & $99 \%$ \\
\hline Next-generation & 10 & 0.5 & 1.0 & $5 \%+10 \% / \sqrt{E}$ & $2+10 / \sqrt{E}$ & 0.1 & 1 & $99 \%$ \\
\hline
\end{tabular}

Table 2: Physical inputs for the response functions of the detectors considered in this study: total mass, threshold and plateau energy for the detection efficiency curve, energy and zenith resolutions, and threshold and plateau energy for the classification efficiency curve.

key performance parameters. We model the detection efficiency (parametrizing the probability for a neutrino event to be successfully detected) by a 2-parameter sigmoid function in $\log (E)$ adjusted to fit predefined threshold and plateau levels corresponding respectively to 5\% and 95\% detection efficiency. The energy resolution is described by a Gaussian pdf with tunable width $\sigma(E) \sim E$. The zenith angle resolution is obtained from a 2D Fisher distribution marginalized over the azimuth; it can be approximated by a Gaussian distribution with tunable width $\sigma(E) \sim \sqrt{E}$. Finally, the classification efficiency (parametrizing the probability for a neutrino event to be correctly identified flavour-wise) is modeled by a 2-parameter sigmoid function in $\log (E)$ adjusted to the $5 \%$ and $95 \%$ classification efficiency.

\section{Results and discussion}

We have used the above approach to investigate the influence of various detector performance indicators on their ability to detect a departure from the default Z/A in the outer core caused by the presence of Hydrogen. To that purpose, we have considered three benchmark detectors whose response functions reflect the expected performances of DUNE, ORCA and Hyperkamiokande, as well as a hypothetical "next-to-next generation" detector that would combine the best characteristics of each. The physical parameters used to build the different detector response functions are detailed in Table 2. We fold these response functions with the underlying signal to obtain the expected event distributions in each of the two channels, for the default model (Fe-Ni alloy) and for model (D) which has the highest Hydrogen content. An event statistics corresponding to 10 years data taking is assumed for all detectors. All results shown here have been obtained under the (favourable) assumption of normal mass ordering of the neutrinos, considering that this measurement will be achieved in a much shorter timescale than the tomography one.

We quantify the expected signal in terms of a signed chi-squared function $\chi^{2}(\operatorname{bin} i)=\left(n_{0}^{i}-\right.$ $\left.n_{D}^{i}\right) \times\left|n_{0}^{i}-n_{D}^{i}\right| / n_{0}^{i}$ (where the subscripts 0 and $\mathrm{D}$ refer to the corresponding compositional models from Table 1), assuming bin-by-bin Poisson statistics. Summing the $\left|\chi_{i}^{2}\right|$ over all bins provides a good estimate of the significance to reject model (0) if model (D) is true (and vice-versa), with $\sum_{i}\left|\chi_{i}^{2}\right|=1$ for $\sim 1 \sigma$ significance. In accordance with the predictions of theoretical underlying signal, we find that the muon neutrino (or track) channel provides a higher sensitivity level than the electron neutrino (or cascade) channel for all three detector benchmarks. As shown in Fig. 4, the three options exhibit comparable statistical significance in the measurement of the outer core compositional model after 10 years of data taking, although their sensitivity does not necessarily come from the same region of the $(E, \theta)$ plane. These plots also illustrate how the detection threshold and energy/zenith resolutions affect the sharpness of the patterns of detected events with respect to the expected signal shown on Fig. 2. 

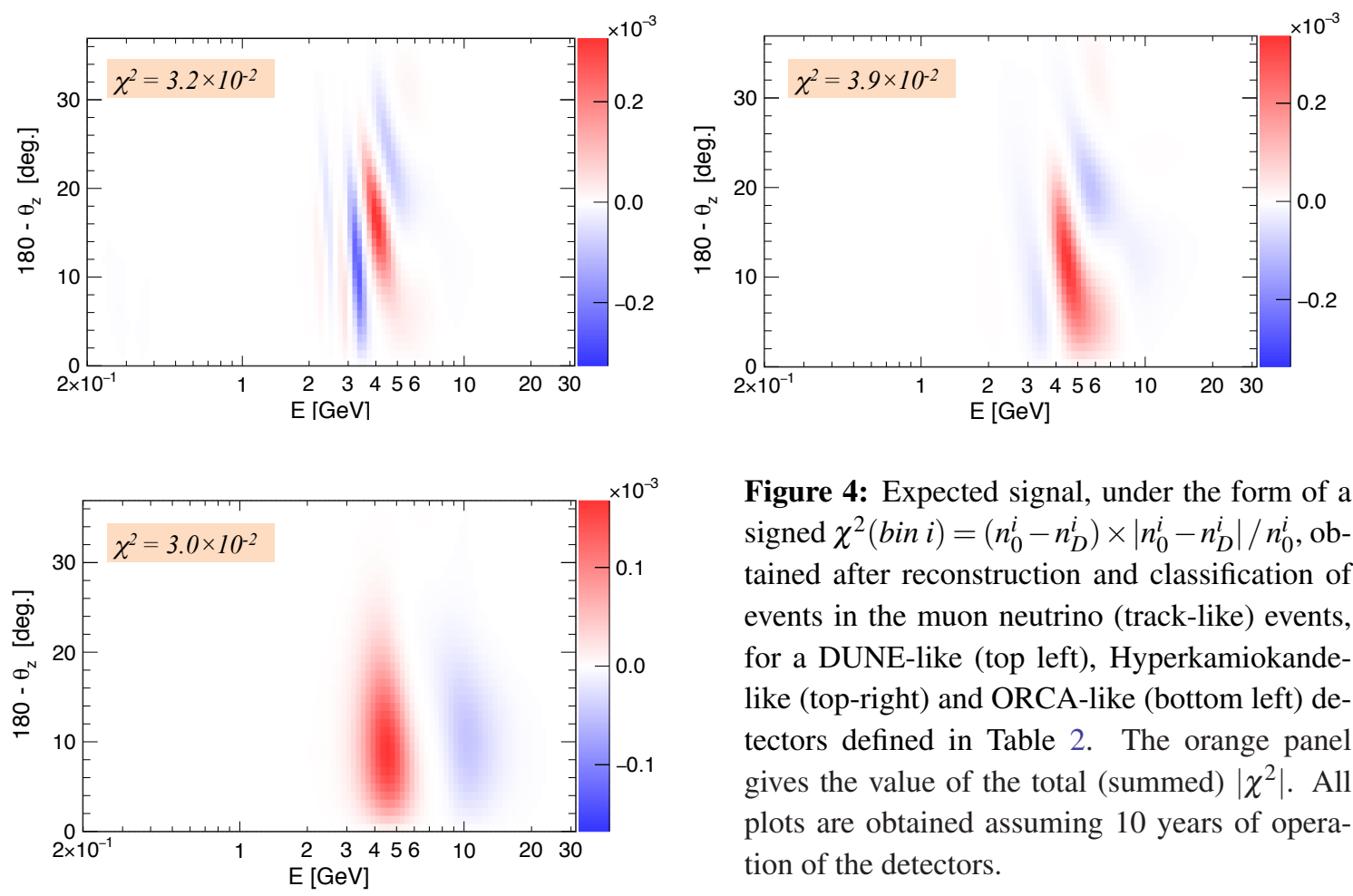

Figure 4: Expected signal, under the form of a signed $\chi^{2}($ bin $i)=\left(n_{0}^{i}-n_{D}^{i}\right) \times\left|n_{0}^{i}-n_{D}^{i}\right| / n_{0}^{i}$, obtained after reconstruction and classification of events in the muon neutrino (track-like) events, for a DUNE-like (top left), Hyperkamiokandelike (top-right) and ORCA-like (bottom left) detectors defined in Table 2. The orange panel gives the value of the total (summed) $\left|\chi^{2}\right|$. All plots are obtained assuming 10 years of operation of the detectors.

We conclude that detectors currently under construction or design (ORCA, DUNE, HyperKamiokande) only have the capability of measuring the amount of $\mathrm{H}$ in the core with a precision of a few percent in weight. To achieve a $1 w t \%$ precision level, a next-generation detector would be needed, that combines characteristics achieved by each of the current detectors, such as a large detection volume with good energy and angular resolutions. Additionally, lowering the detection threshold to the $1 \mathrm{GeV}$ level with reasonably good flavour identification performance in the few-GeV region is critical. Fig. 5 illustrates the performance in compositional model separation expected for such a next-generation detector, expressed in terms of event number differences, in the muon-neutrino (track-like) channel. In such a case, the compositional model (D) with $1 \mathrm{wt} \%$ hydrogen could be tested at $1 \sigma$ confidence level, provided that systematic uncertainties (not yet included in this study) can be kept under control. The liquid Argon technology being unlikely scalable to the $\sim 10$ Mton scale, a most promising alternative might reside in a large-scale densified water Cherenkov detector in the line of the recently proposed Super-ORCA detector [22].

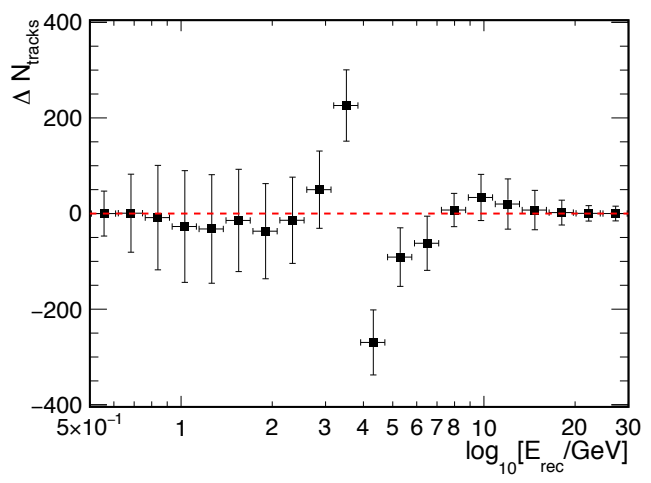

Figure 5: Expected signal, in terms of the mean difference of number of events

$$
\Delta N_{\text {tracks }}=\left\langle n_{0}-n_{D}\right\rangle
$$

and measurement errors for neutrinos measured with $\theta<20^{\circ}$ in the muon-neutrino (track-like) channel, for a next-generation detector with characteristics as in Table 2. 


\section{Acknowledgements}

The authors gratefully acknowledge financial support from LabEx UnivEarthS at Sorbonne Paris Cité (ANR-11-IDEX-0005-02, ANR-10-LABX-0023) and Université de Paris (ANR-18IDEX-0001) and from the Agence Nationale de la Recherche (ANR-15-CE31-0020).

\section{References}

[1] M. Dziewonski and D. L. Anderson, Physics of the Earth and Planetary Interiors 25297 (1981).

[2] F. Birch, J. Geophys. Res., 57(2), 227-286 (1952).

[3] J.-P. Poirier, Phys. Earth Planet. Inter. 85, 319-337 (1994); K. Hirose, S. Labrosse, and J. Hernlund (2013), Annu. Rev. Earth Planet Sci., 41, 657-691.

[4] J. Badro et al., Proc. Natl. Acad. Sci. U S A., 112(40): 12310-12314 (2015).

[5] T. Sakamaki et al., Earth and Planetary Science Letters 287 293-297 (2009).

[6] E. Ohtani et al., Phys. Chem. Minerals 78 77-82 (2005).

[7] K.Umemoto and K.Hirose, Geophys. Res. Lett. 42(18), 7513-7520 (2015); T. Sakamaki et al., Science Advances 2(2), e15008022016 (2016).

[8] see e.g. the review by W. Winter, Earth Moon Planets 99 285-307 (2006).

[9] S. Mikheev \& A. Smirnov, Sov. J. Nucl. Phys. 42913 (1985); L. Wolfenstein, Phys. Rev. D 172369 (1978); S. Petcov, Phys. Lett. B434 321 (1998); M. Chizhov \& S. Petcov, Phys. Rev. D63 073003 (2001).

[10] C. Rott, A. Taketa \& D. Bose, Sci.Rep. 515225 (2015); W. Winter, Nucl.Phys. B908 250-267 (2016).

[11] M. Honda et al., Phys. Rev. D92 023004 (2015); we have used the tables for Gran Sasso site without mountain, azimuth-averaged, at solar minimum.

[12] https://github.com/joaoabcoelho/OscProb

[13] C. Andreopoulos et al., Nucl. Instrum. Meth. A614 87-104 (2010).

[14] https://earthref.org/GERMRD/datamodel/ (cit. on pp. 114, 115).

[15] E. Kaminski and M. Javoy, Earth Plan. Sci. Lett. 365 97-107 (2013).

[16] S. Tagawa et al., Geophys. Res. Lett. 43(8) 3686-3692 (2016).

[17] The Hyper-Kamiokande Proto-collaboration, Hyper-Kamiokande design report, e-Print:arXiv:1805.04163v2 [physics.ins-det]

[18] The KM3NeT Collaboration, J. Phys. G 43(8), 084001 (2016); B. Strandberg [KM3NeT Coll.], PoS(ICRC2019) 1019.

[19] The IceCube Collaboration, J.Phys. G 44(5), 054006 (2017);

[20] The DUNE Collaboration, FERMILAB-DESIGN-2016-04, e-Print: arXiv:1601.02984; FERMILAB-DESIGN-2018-02, e-Print: arXiv:1807.10334 .

[21] S. Bourret et al. [KM3NeT Coll.], PoS(ICRC2017) 1020; EPJ Web Conf. 20704008 (2019).

[22] J. Hofestädt, M. Bruchner and T. Eberl, PoS(ICRC2019) 911. 